\title{
Combination of gambogic acid with cisplatin enhances the antitumor effects on cisplatin- resistant lung cancer cells by downregulating MRP2 and LRP expression
}

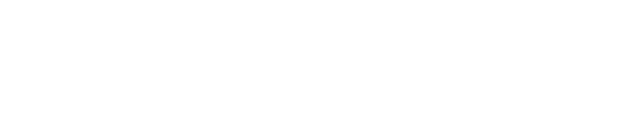

\section{Wendian Zhang* \\ Hechao Zhou* \\ Ying Yu* \\ Jingjing $\mathrm{Li}$ \\ Haiwen Li \\ Danxian Jiang \\ Zihong Chen \\ Donghong Yang \\ Zumin Xu \\ Zhonghua Yu}

Cancer Center, Affiliated Hospital of Guangdong Medical University,

Zhanjiang, Guangdong Province,

People's Republic of China

*These authors contributed equally to this work
Correspondence: Zhonghua Yu;

Zumin Xu

Cancer Center, Affiliated Hospital of Guangdong Medical University, 57 South Renmin Road, Zhanjiang 524000 Guangdong Province, People's Republic of China

Tel +86759 $23878 \mid 3 ;+867592387448$ Fax +86759238 78। 3; +867592387448 Email zhonghua_yu@।26.com; zuminxu@I63.com
Abstract: Cisplatin resistance is a main clinical problem of lung cancer therapy. Gambogic acid (GA) could prohibit the proliferation of a variety of human cancer cells. However, the effects of GA on cisplatin-resistant lung cancer are still unclear. The objective of the present study was to find out the antitumor effects of GA on cisplatin-resistant human lung cancer A549/DDP cells and further explore its underlying mechanisms. Cell Counting Kit-8 assay was used to observe the impacts of GA and/or cisplatin on the proliferation of lung cancer cells; flow cytometry was used to detect the effects of GA on cell cycle and apoptosis; Western blot was used to examine the effects of GA on the expression of lung resistance protein (LRP) and multidrug resistanceassociated protein 2 (MRP2) protein in A549/DDP cells. Our results showed that GA dose- and time-dependently prohibited the proliferation and induced significant cell apoptosis in A549 and A549/DDP cells. GA also induced G0/G1 arrest in both A549/DDP and A549 cells. Moreover, GA upregulated protein expression level of cleaved caspase-3 and Bax and downregulated protein expression level of pro-caspase- 9 and Bcl-2 in time- and dose-dependent way in A549/ DDP cells. GA combined with cisplatin enhanced the cells apoptotic rate and reduced the cisplatin resistance index in A549/DDP cells. In addition, GA reduced the MRP2 and LRP protein expression level in A549/DDP cells. GA inhibits the proliferation, induces cell cycle arrest and apoptosis in A549/DDP cells. Combination of GA with cisplatin enhances the antitumor effects on cisplatin-resistant lung cancer cells by downregulating MRP2 and LRP expression.

Keywords: gambogic acid, cisplatin, drug resistance, lung cancer, lung resistance protein, multidrug resistance-associated protein 2, anti-tumor, cell cycle arrest, cell apoptosis

\section{Introduction}

Lung cancer is the commonest cause of cancer-related death around the world. Non-small-cell lung cancer (NSCLC) occupies $\sim 75 \%-80 \%$ of lung cancer. ${ }^{1}$ Platinumbased doublet chemotherapeutic regimens have become the primary therapeutic method for NSCLC. However, the 5-year survival rate is still very low. ${ }^{2}$ Cisplatin resistance is a main clinical problem of lung cancer therapy. ${ }^{3}$ Therefore, it is urgently needed to find novel agent that possess anticancer effects on the cisplatin-resistant lung cancer cells.

Gambogic acid (GA) is a main ingredient of gamboge, the resin secreted from Garcinia hanburyi tree in South-East Asia. ${ }^{4} \mathrm{GA}$ has been used as one of traditional medicine for centuries and it possesses many kinds of biological effects such as anti-infectious, anti-oxidant, anti-inflammatory, and antiviral. ${ }^{5}$ In recent years, many researchers have found that GA could inhibit the proliferation of a variety of human cancer cells in vitro, 
including lung cancer, ${ }^{6}$ leukemia, ${ }^{7}$ colorectal cancer, ${ }^{8}$ prostate cancer, ${ }^{9}$ hepatocarcinoma, ${ }^{10}$ and breast cancer. ${ }^{11}$ The possible antitumor mechanisms of GA are associated with the enhancement of reactive oxygen species accumulation, ${ }^{8}$ inhibition of telomerase, ${ }^{12}$ induction of apoptosis, ${ }^{13}$ and interruption of nuclear factor- $\mathrm{\kappa B}$ signal pathway. ${ }^{14}$ Also, GA exhibits anticancer effects on NCI-H1993 xenografts by regulation of the MET signal pathway. ${ }^{15} \mathrm{GA}$ is a prospective antitumor drug with less toxic effects on the normal tissues, ${ }^{16}$ which has been authorized for the treatment of variety of cancer in clinical trials by the Chinese Food and Drug Administration. ${ }^{17}$ A Phase IIa clinical study suggests that GA administered at $45 \mathrm{mg} / \mathrm{m}^{2}$ is safe. ${ }^{18}$ Moreover, GA could enhance antitumor effects of cisplatin on both osteosarcoma ${ }^{19}$ and lung cancer. ${ }^{20}$ In addition, GA not only could sensitize esophageal cancer cells to radiation via blocking Akt/mammalian target of rapamycin pathway and nasopharyngeal carcinoma cells in hypoxic conditions, ${ }^{21,22}$ but also reverse docetaxel resistance in gastric cancer, ${ }^{23}$ doxorubicin resistance in breast cancer ${ }^{24}$ and ovarian cancer, ${ }^{25} 5$-fluorouracil resistance in colorectal cancer, ${ }^{13}$ and the multidrug resistance of human in epithelial cancer. ${ }^{26}$

However, the impact of GA on cisplatin-resistant lung cancer is still unknown. The purpose of this study was to find out effects of GA in aspect of cell growth, cell cycle, and apoptosis and observe the antitumor effects of GA combined with cisplatin in cisplatin-resistant lung cancer A549/DDP cells and explore its underlying mechanisms.

\section{Materials and methods}

\section{Reagents}

GA ( $>98 \%$ purity, Sigma-Aldrich, St Louis, MO, USA) was soluble in dimethyl sulfoxide and conserved at $-20^{\circ} \mathrm{C}$. The preservation solution was diluted to different concentrations in use, in which the dimethyl sulfoxide concentration was $<0.1 \%$. Cisplatin was purchased from Stockhausen Pharmaceutical Co., Ltd (Lianyungang, Jiangsu, People's Republic of China). Roswell Park Memorial Institute medium 1640 was obtained from HyClone (Logan, UT, USA). Cell Counting Kit-8 (CCK-8) was acquired from Dojindo (Rockville, MD, USA). Propidium iodide (PI), RNase A, and annexin V-fluorescein isothiocyanate was purchased from Keygen Biotechnology (Nanjing, Jiangsu, People's Republic of China) and dissolved in phosphate buffered saline (PBS). The primary antibodies against Bcl-2, Bax, anticaspase-9, anticaspase-3, multidrug resistance-associated protein 2 (MRP2), lung resistance protein (LRP), and $\beta$-tubulin were acquired from Cell Signaling Technology (Danvers, MA, USA). And goat antimouse or antirabbit IgG-HRP secondary antibody was acquired from Beyotime Biotech (Nanjing, Jiangsu, People's Republic of China).

\section{Cell culture}

Human lung adenocarcinoma cisplatin-resistant cell lines (A549/DDP) and cisplatin-sensitive cell lines (A549) were acquired from BioLeaf Biotech (Shanghai, People's Republic of China) and American Type Culture Collection (Manassas, VA, USA) and cultured in Roswell Park Memorial Institute medium 1640 with 10\% fetal bovine serum (FBS; Biological Industries, Cromwell, CT, USA), maintained in a suitable environment with $5 \% \mathrm{CO}_{2}$ at $37^{\circ} \mathrm{C}$. This study was performed with the approval of the Institutional Ethic Committee of Guangdong Medical University.

\section{Cell proliferation assay}

A549 and A549/DDP cells were seeded in a 96-well plate at density of $5 \times 10^{3}$ cells per well. Incubated $\sim 24$ hours later, the former medium was replaced by fresh medium with GA as following concentrations $(0,0.5,1.0,1.5,2.0,2.5$, and $3.0 \mu \mathrm{M}$ ) for 24 and 48 hours in the presence of $1 \%$ FBS. Cell viability was tested by using the CCK- 8 assay. The cells were incubated $\sim 2$ hours before absorbance read for the CCK-8 proliferation assay. Each well absorbance was tested at $450 \mathrm{~nm}$ by microplate reader. The proliferation rate was defined in terms of the percentage of each group surviving cells compared with the untreated group.

\section{Cell cycle analysis}

For flow cytometry analysis, $2 \times 10^{5}$ cells were gathered by $1 \times 10^{3} \mathrm{r} / \mathrm{min}$ centrifugation following GA treatment $(0,1.0$, $2.0 \mu \mathrm{M}$ ) for 24 hours at $37^{\circ} \mathrm{C}$. The cells were fixed with $70 \%$ ethanol at $4^{\circ} \mathrm{C}$ overnight after washing it twice with PBS. The fixed cells were washed one time with PBS and incubated with $50 \mu \mathrm{g} / \mathrm{mL}$ PI plus $100 \mu \mathrm{g} / \mathrm{mL}$ RNase A before the flow cytometry analysis.

\section{Cell apoptosis assay}

For flow cytometry analysis, $1 \times 10^{5}$ cells were harvested after different treatment, collected by centrifugation $(10 \mathrm{~min}$, $1 \times 10^{3} \mathrm{r} / \mathrm{min}$ ) and washed with PBS three times. The cells were resuspended in binding buffer then incubated with $5 \mu \mathrm{L}$ annexin V-fluorescein isothiocyanate and PI for 15 minutes in the dark before analyzing by flow cytometry.

\section{Resistance index of cisplatin}

The calculation way we learned from this article. ${ }^{27}$ The A549/ DDP cells cisplatin resistance index (RI) was also tested by 
the CCK-8 assay. A549/DDP cells and A549 were seeded in 96-well plates at density of $5 \times 10^{3}$ cells per well. Incubated $\sim 24$ hours later, the former medium was replaced by fresh medium with cisplatin at the following concentrations $(0,2.5,5.0,10,20$, and $40 \mu \mathrm{g} / \mathrm{mL})$ for 24 and 48 hours in the presence of $1 \%$ FBS. Cells were prepared for absorbance reading after 24 and 48 hours incubation. Cell inhibitory rate was calculated using the formula: inhibitory rate $=100 \times$ $\left(A_{\text {con }}-A_{\mathrm{t}}\right) / A_{\text {con }}$, where $A_{\text {con }}$ is the A value of the control group and $A_{\mathrm{t}}$ is the $A$ value of the treatment group. The $50 \%$ inhibitory concentration $\left(\mathrm{IC}_{50}\right)$ was calculated from three independent experiments' dose-response data. The A549/ DDP cells RI of cisplatin was calculated using the formula: $\mathrm{RI}=\mathrm{IC}_{50} \mathrm{~A} 549 / \mathrm{DDP} / \mathrm{IC}_{50} \mathrm{~A} 549$. The experiment was repeated in the presence of $2 \mu \mathrm{M}$ GA.

\section{Western blot analysis}

Cells were seeded in six-well plates at a density of $1 \times 10^{5}$ per well. After treatment with GA and cisplatin, the preparation of total protein samples from the culture cells for immunoblotting was carried out as previously described. ${ }^{28}$ The membrane was incubated with the following indicated antibody: Bax, Bcl-2, caspase-3, caspase-9, MRP2, LRP, and $\beta$-tubulin. The interest protein was tested with goat antimouse or antirabbit IgG-HRP secondary antibody.

\section{Statistical analysis}

The data were expressed as mean \pm standard deviation. Student's $t$-test method was used for two-group comparison. One-way ANOVA was used for more than two-group comparison. All statistical analyses were calculated by SPSS 13.0 software (SPSS, Chicago, IL, USA). $P<0.05$ was considered to be statistically significant.

\section{Results}

\section{GA prohibits the growth of A549/DDP and A549 cells}

Both A549/DDP and A549 cells were exposed to different concentrations of GA $(0,0.5,1.0,1.5,2.0,2.5$, and $3.0 \mu \mathrm{M})$ for 24 and 48 hours. Cell viability was tested by CCK-8 assay. The results showed that GA prohibited A549/DDP and A549 cells proliferation in a time- and dose-dependent manner (Figure 1, $P<0.05$ ). The $\mathrm{IC}_{50}$ after GA treatment for 24 hours in the A549/DDP and A549 cells were 2.591 \pm 0.782 and $2.261 \pm 0.218 \mu \mathrm{M}$, respectively.

\section{GA induces cell cycle arrest at G0/GI in A549 and A549/DDP cells}

To figure out whether GA inhibits cell proliferation by promoting cell cycle progression changes, flow cytometry was used to detect the impact of GA on cell cycle. A549 and A549/ DDP cells were exposed to $0,1.0$, and $2.0 \mu \mathrm{M}$ GA for 24 hours. The results indicated that GA caused an increase in the G0/ G1 stages in A549/DDP and A549 cells, suggesting that GA induced G0/G1 cell cycle arrest (Figure 2A and B, $P<0.01$ ).

\section{GA induces A549/DDP cells apoptosis in time- and dose-dependent way}

A549/DDP cells were treated with $0,0.5,1.0$, and $2.0 \mu \mathrm{M}$ GA for 24 hours and $2 \mu \mathrm{M}$ GA for $24-72$ hours. After GA
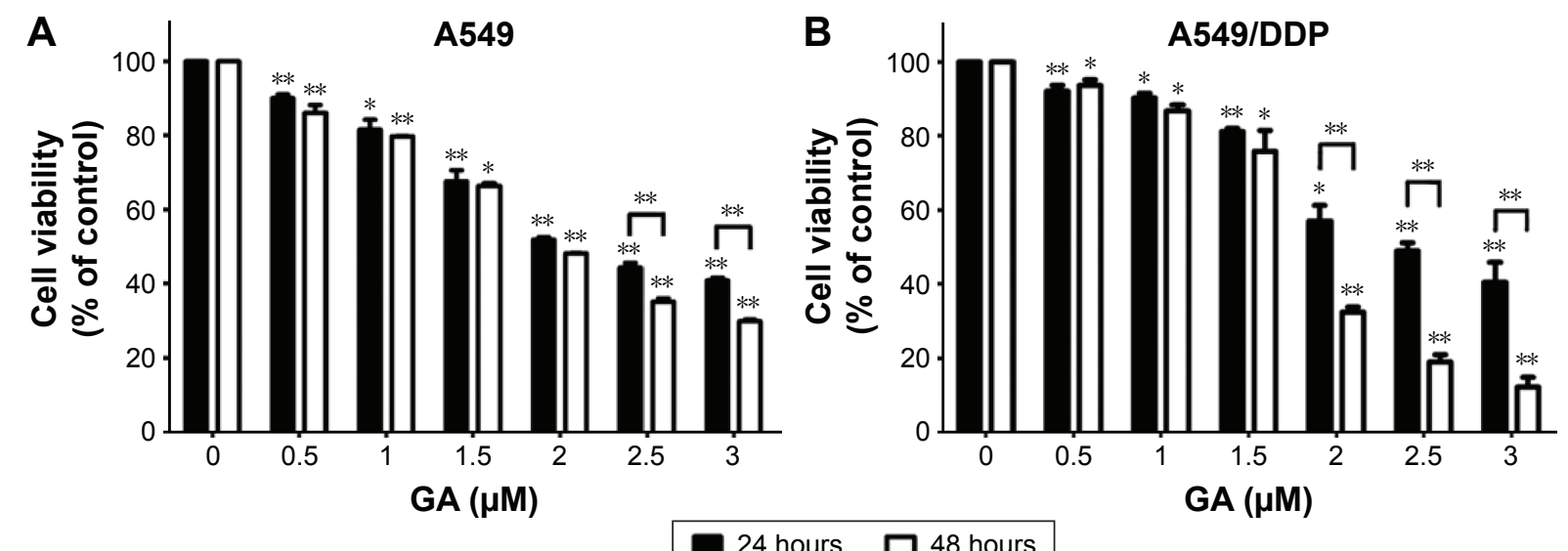

24 hours 48 hours

Figure I GA inhibits the growth of A549 (A) and A549/DDP (B) cells.

Notes: Human lung adenocarcinoma cells were treated with GA at the indicated concentrations $(0,0.5,1.0,1.5,2.0,2.5$, and $3.0 \mu \mathrm{M})$ for 24 and 48 hours, and the proliferation rates were tested by CCK-8 assay. GA significantly reduced the viability of the two cell lines in a dose- and time-dependent manner. All data are presented as the mean values \pm SD from three independent experiments. Cell proliferation in the untreated control cells was regarded as $100 \%$. $* P<0.05$; $* * P<0.0 \mathrm{I}$ vs control cells; bars, SD.

Abbreviations: CCK-8, Cell Counting Kit-8; GA, gambogic acid; SD, standard deviation. 
A

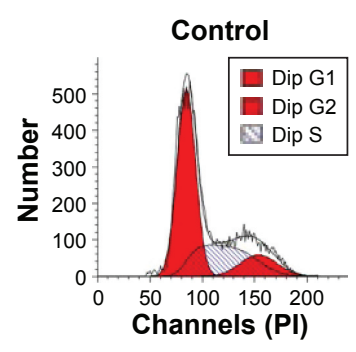

B

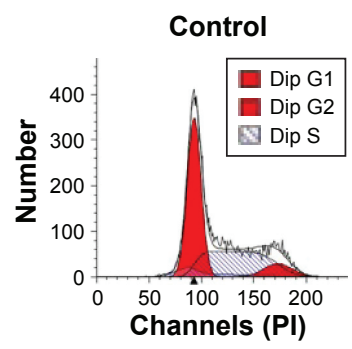

A549

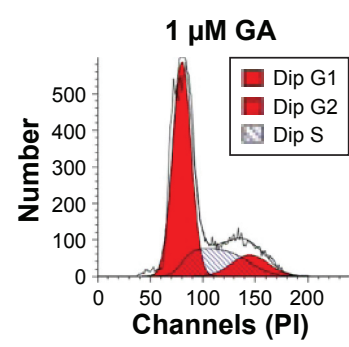

A549/DDP

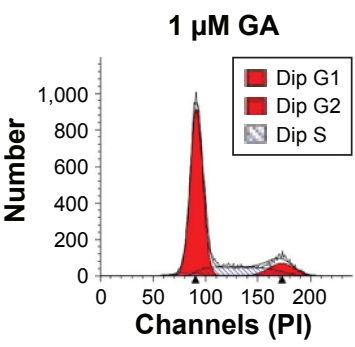

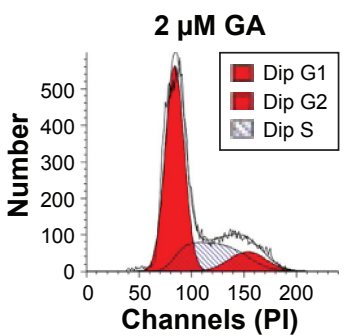

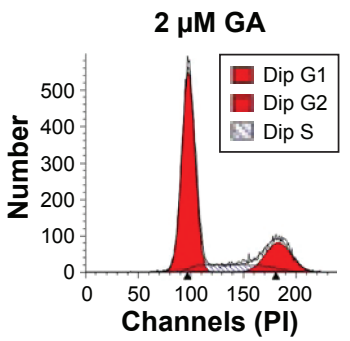

A549 24 hours

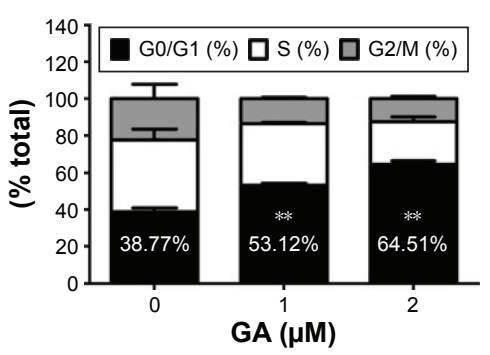

A549/DDP 24 hours

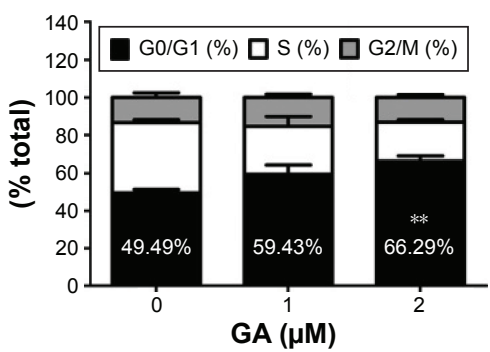

Figure 2 GA induces G0/GI cell cycle arrest in A549 (A) and A549/DDP (B) cells.

Notes: A549 and A549/DDP cells were treated with $0,1.0$, and $2.0 \mu \mathrm{M}$ GA for 24 hours. After staining with propidium iodide, cell cycle stage was analyzed by flow cytometry. The cell cycle distribution was quantified in GA-treated and -untreated control cells. All data are presented as the mean \pm SD of at least three independent experiments. ${ }^{*} P<<0.01$ vs control cells; bars, $S D$.

Abbreviations: GA, gambogic acid; SD, standard deviation; PI, propium iodide.

treatment at $0.5,1.0$, and $2.0 \mu \mathrm{M}$ for 24 hours, the cells apoptosis rate was $14.15 \% \pm 7.70 \%, 20.18 \% \pm 7.91 \%$, and $27.30 \% \pm 7.69 \%$, respectively, which were significantly different from the unexposed group $(5.67 \% \pm 2.54 \%$ ) (Figure $3 \mathrm{~A}$, $P<0.05)$. The GA treatment caused an increase in A549/ DDP cells apoptosis for 24-72 hours (Figure 3B, $P<0.05$ ). The results illustrated that GA could induce A549/DDP cells apoptosis in a time- and dose-dependent way. To further ensure that caspase activation was related to GAinduced A549/DDP cells apoptosis process, we found that GA upregulated the protein expression level of cleaved caspase- 3 and Bax and downregulated protein expression level of pro-caspase-9 and Bcl-2 in A549/DDP cells (Figure 3C-F, $P<0.01$ ).

\section{GA reduces the resistance index of A549/DDP cells}

Cell proliferation inhibited by cisplatin is apparently attenuated in A549/DDP cells compared with A549 cells. However, the sensitivity of A549/DDP cells with the presence of GA to cisplatin is more than absence of GA. In addition, cisplatin RI of the A549/DDP cells was significantly weakened by the GA and the reversal multiples was 1.81 (24 hours) and 2.05 ( $48 \mathrm{~h}$ ) (Figure 4, $P<0.01$ ). Therefore, the data indicated that GA dramatically reduced the A549/DDP cells cisplatin resistance.

\section{GA combined with cisplatin results in an} enhancement of cell apoptosis effects in a time-dependent way

The A549/DDP cells were treated with a combination of cisplatin $(10 \mu \mathrm{g} / \mathrm{mL})$ and $\mathrm{GA}(2 \mu \mathrm{M})$. After treatment of GA and cisplatin for 24-72 hours, the apoptotic rate of A549/ DDP cells were $18.0 \%, 52.4 \%$, and $74.8 \%$, respectively. While the control and monotreatment groups were less than combined treatment group (Figure 5A, $P<0.01$ ). Meanwhile, GA and/or cisplatin induced A549/DDP cell apoptosis in a time-dependent way. Furthermore, after the same treatment Western blot was used to detect the expression of Bcl-2, Bax, caspase-3, and caspase-9 in the A549/DDP cells. The results showed that the combination of these two drugs caused a significantly greater increase in cleaved caspase- 3 and Bax and decrease in pro-caspase- 9 and Bcl-2 than either agent alone (Figure 5B-F, $P<0.01$ ). Taken together, these results showed that GA combined with cisplatin could be more efficient via apoptosis-inducing action on A549/DDP cells.

\section{GA downregulates the protein expression of MRP2 and LRP in a dose- dependent way}

To further figure out the underlying mechanisms of GA reducing A549/DDP cells resistance index, we first examined the basic expression level of MRP2 and LRP protein in 
A

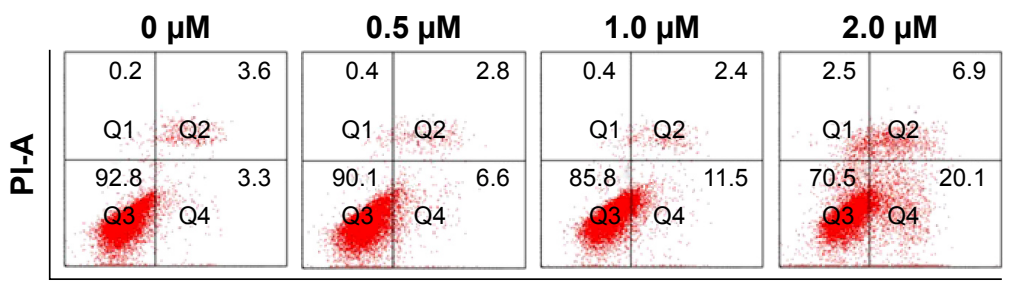

FITC-A

B

\begin{tabular}{|c|c|c|c|c|c|c|c|}
\hline \multicolumn{2}{|c|}{ Control } & \multicolumn{2}{|c|}{24 hours } & \multicolumn{2}{|c|}{48 hours } & \multicolumn{2}{|c|}{72 hours } \\
\hline 0.2 & 1.1 & 0.4 & 8.8 & 1.0 & 8.8 & 1.1 & 14.0 \\
\hline Q1 & Q2 & Q1 & $Q 2$ & Q1 & & Q1 & Q2. \\
\hline 97.7 & 0.9 & 81.6 & 9.2 & 59.5 & 30.8 & & 37.3 \\
\hline & Q4 & & Q4 & & & & Q4 \\
\hline
\end{tabular}

FITC-A

C
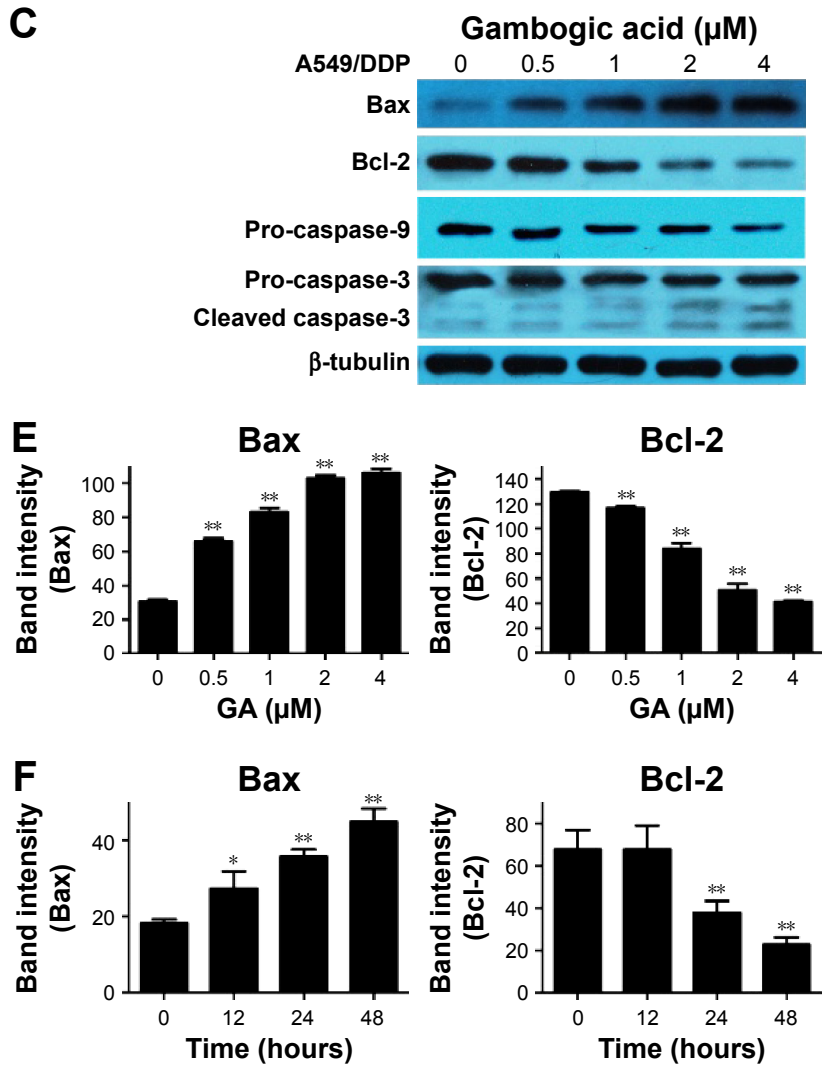

Bcl-2

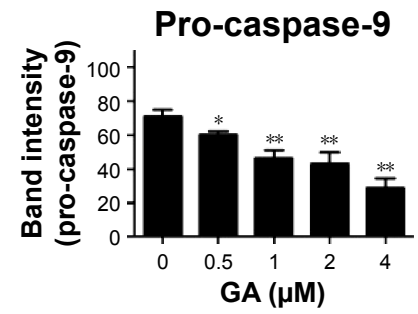

GA $(\mu \mathrm{M})$

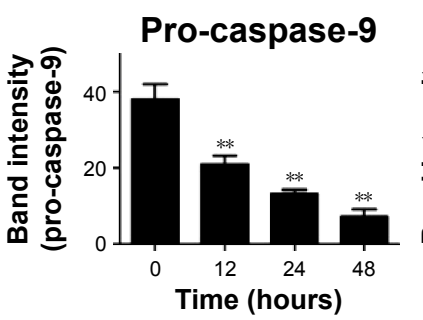

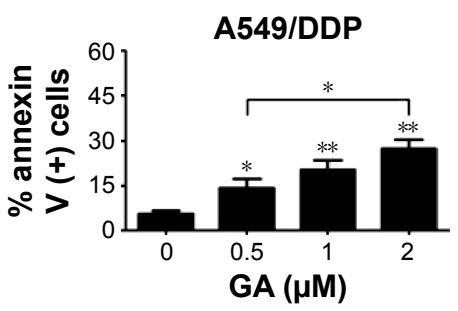

A549/DDP

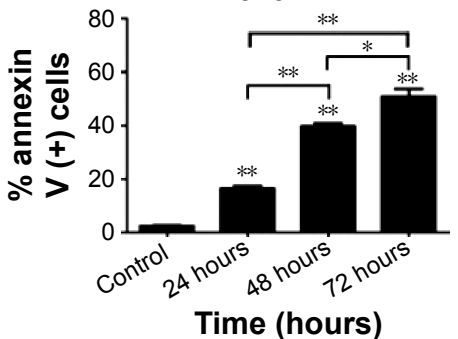

Time (hours)

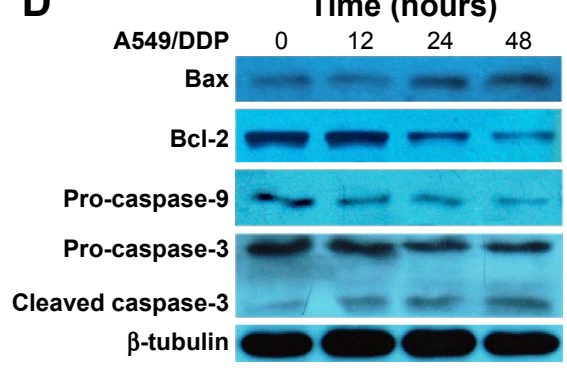

Figure $3 \mathrm{GA}$ induces apoptosis in A549/DDP cells in a dose- and time-dependent way.

Notes: (A) A549/DDP cells were treated with 0, 0.5, 1.0, and $2.0 \mu \mathrm{M}$ GA for 24 hours. (B) A549/DDP cells were treated with $2 \mu \mathrm{M}$ GA for $0,24,48$, and 72 hours. Cell apoptosis was analyzed by flow cytometry at various time points. The cell apoptotic rates were quantified. (C) A549/DDP cells were treated with GA at the indicated concentrations $(0,0.5,1.0,2.0$, and $4.0 \mu \mathrm{M})$ for 24 hours. (D) A549/DDP cells were treated with GA for 0, I2, 24, and 48 hours. The levels of Bax, Bcl-2, caspase-9, and caspase- 3 were determined by Western blot analysis. (E and F) GA upregulated the expression level of Bax and cleaved caspase-3 and downregulated Bcl-2 and pro-caspase- 9 expression in A549/DDP cells. Data are shown as the mean values \pm SD from three independent experiments. $* P<0.05 ; * * P<0.01$; bars, SD.

Abbreviations: GA, gambogic acid; SD, standard deviation.

A549/DDP and A549 cells. The basic protein expression of MRP2 and LRP of A549/DDP cells was significantly more than A549 cells (Figure 6A). We further detected the changes of MRP2 and LRP protein after disposing with GA $(0,0.5$,
1.0, 2.0, $4.0 \mu \mathrm{M})$ for 24 hours. The results showed that A549/ DDP cells in response to GA had a distinct downregulation of protein expression level of MRP2 and LRP compared with untreated cells (Figure 6B, $P<0.01$ ). 

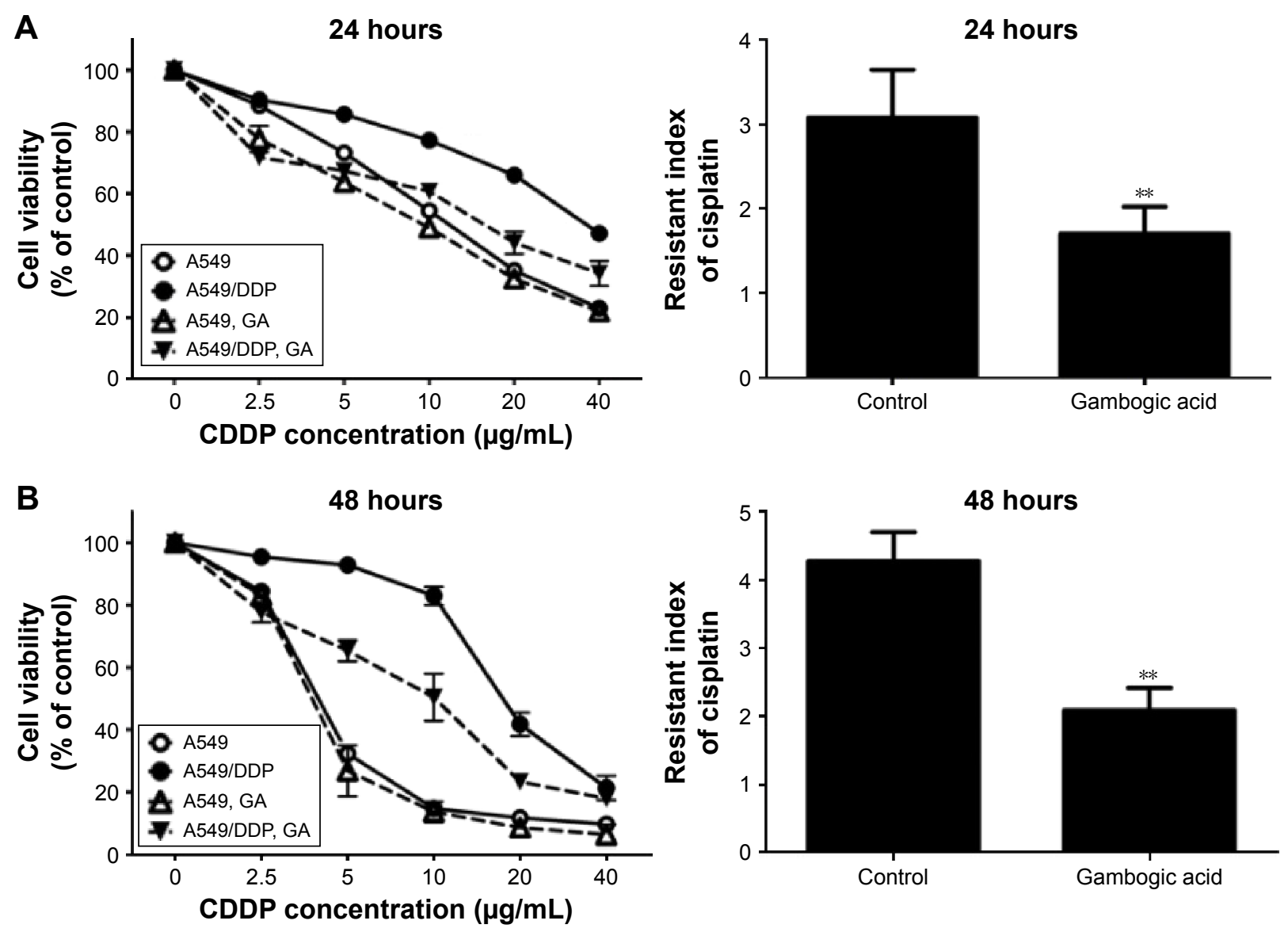

Figure 4 GA reduces the RI of A549/DDP cells. A549 and A549/DDP cells were exposed to increasing concentrations of cisplatin for 24 and 48 hours in the presence or absence of GA $(2 \mu \mathrm{M})$. CCK-8 assay was used to detect cell growth. The rate of growth inhibition in A549 and A549/DDP cell line in the presence and absence of GA for 24 hours (A) and 48 hours (B). RI of cisplatin in A549/DDP cells in the presence and absence of GA was presented at the right side. Values are expressed as mean \pm SD $(n=3)$. Data were analyzed by Student's $t$-test. $* * P<0.01$ vs control cells; bars, SD.

Abbreviations: CCK-8, Cell Counting Kit-8; GA, gambogic acid; RI, resistance index; SD, standard deviation; CDDP, cisplatin.

\section{Discussion}

Resistance to cisplatin-based chemotherapy is still one of the main obstacles in the therapy for lung cancer. ${ }^{29}$ Hence, it is necessary to find novel drug to overcome cisplatin resistance. In this research, we reported for the first time that GA could effectively suppress the proliferation of A549/DDP cells in a time- and dose-dependent way (Figure 1A). Intriguingly, A549 cells showed a higher viability rate after GA treatment at 2, 2.5, and $3 \mu \mathrm{M}$ for 48 hours when compared with A549/DDP cells (Figure 1B), although the conclusion was without too much influence, but which is an unexpected phenomenon worthy of further study. We suppose that cisplatin resistance A549/DDP cells may have some different expression levels of gene or protein compared with A549 cells, which make A549/DDP cells sensitive to GA. Maybe the counterbalance phenomenon of A549/DDP will provide another perspective to solve the problem of cisplatin resistance of lung cancer. Further investigation demonstrated that GA might exert its growth inhibition impact via inducing cell cycle arrest at $\mathrm{G0} / \mathrm{G} 1$ stage (Figure 2B) and cell apoptosis
(Figure 3A and B) on A549/DDP cells. One of the limitations of this research was not to explain this phenomenon of cell cycle arrest by GA on A549/DDP and A549 cells.

To elucidate whether GA could reverse cisplatin resistance in lung cancer, we explored the effects of GA combined cisplatin on A549/DDP cells. Our results showed that cisplatin resistance index of the A549/DDP cells was significantly attenuated by GA and the reversal multiples were 1.81 (24 hours) and 2.05 (48 hours) (Figure 4), which implied that GA may enhance the antitumor effects of cisplatin on cisplatin-resistant lung cancer cells. Similar to this article, other studies also reported that GA could enhance K562/ A02 cells' sensitivity to adriamycin with a reversal multiple of $1.53 .{ }^{30}$ Furthermore, the apoptotic rate was higher when combining GA with cisplatin in comparison with single agent in A549/DDP cells (Figure 5). Consequently, we came to a conclusion that GA could enhance the cytotoxic effects of cisplatin in cisplatin-resistant lung cancer.

Previous studies have suggested that several mechanisms could lead to cisplatin resistance, including reduced 
A
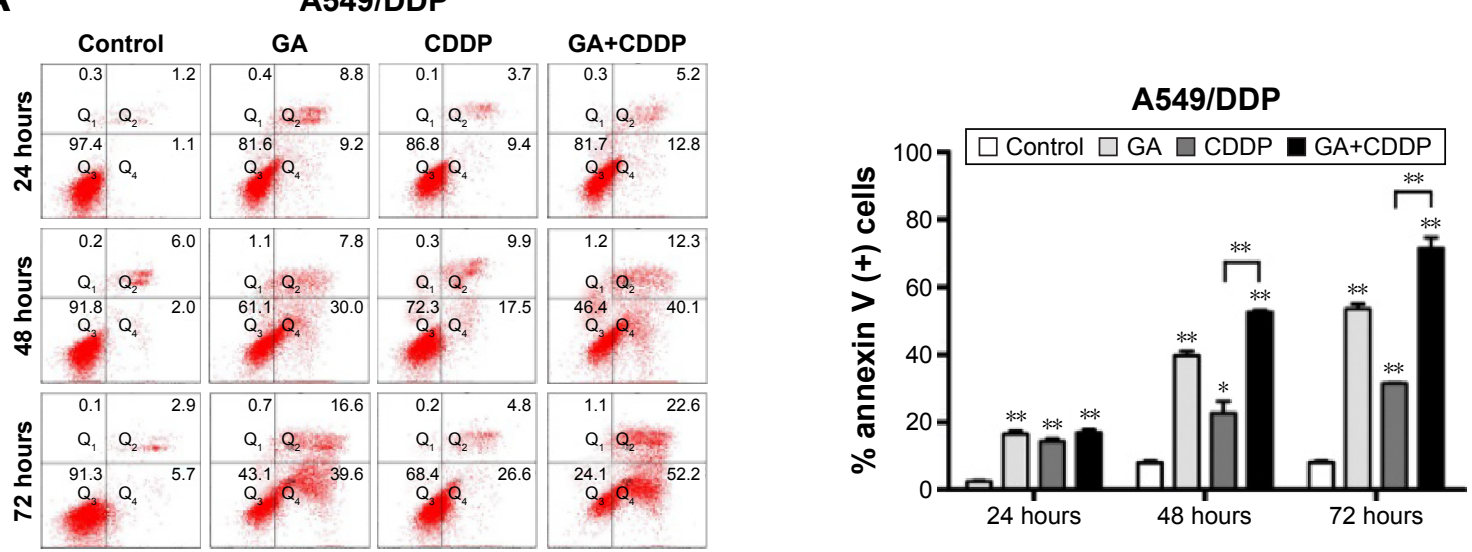

B

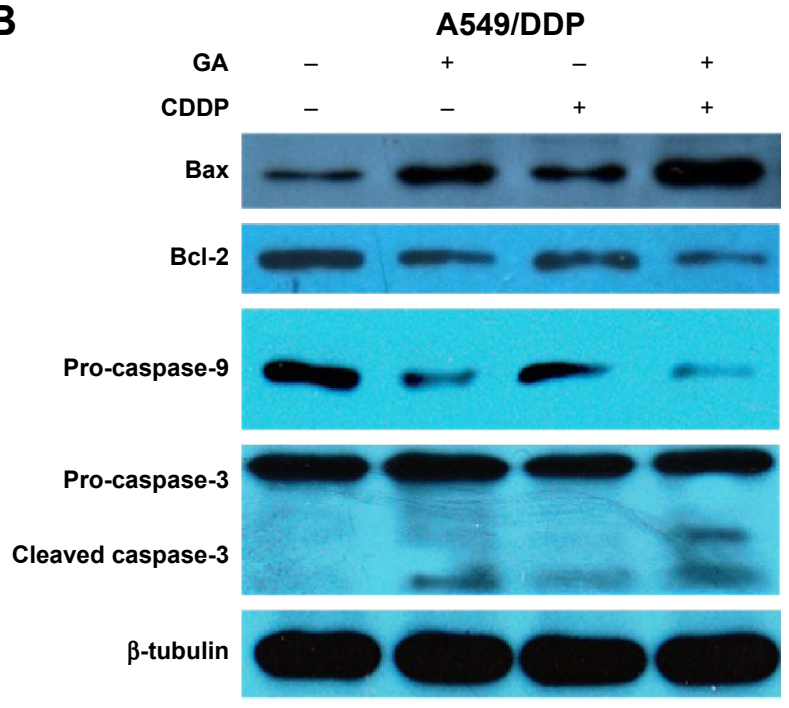

E

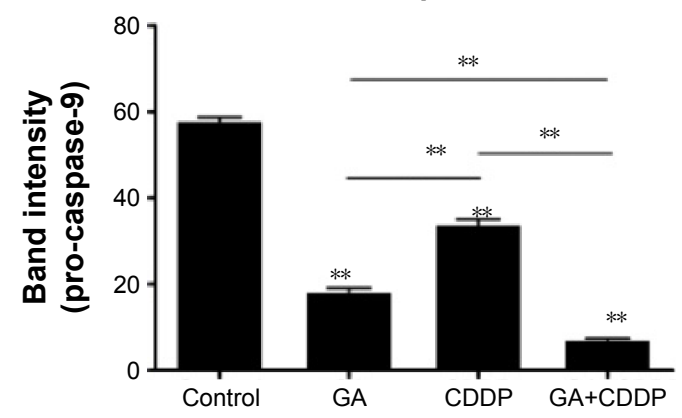

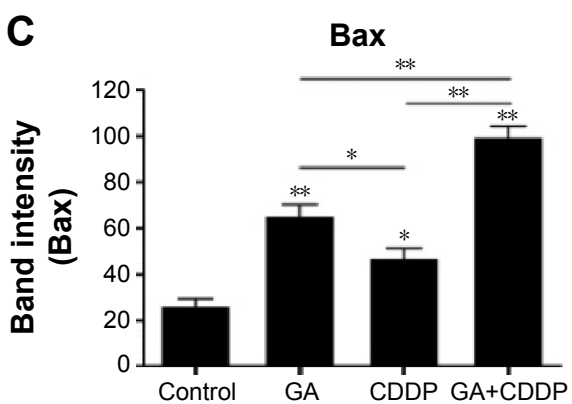

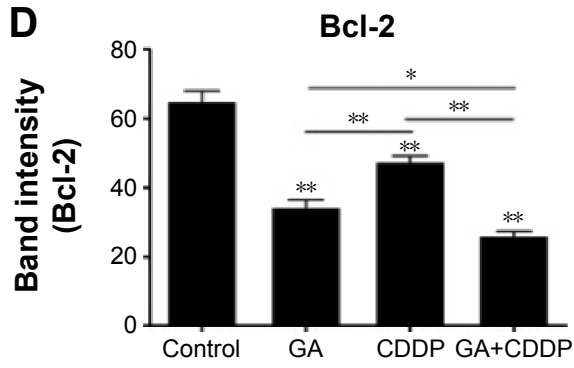

$\mathbf{F}$ Cleaved caspase-3

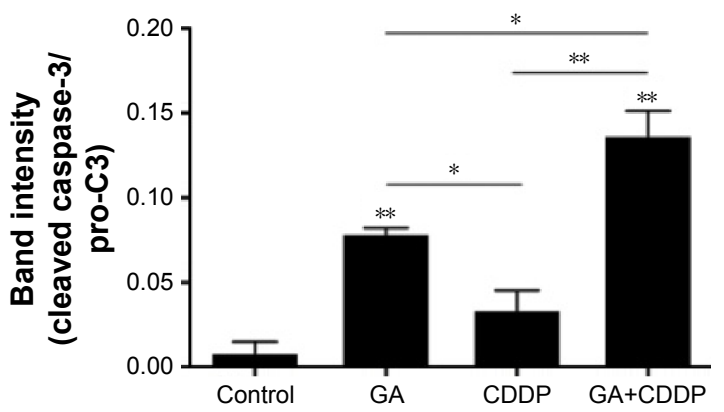

Figure 5 Combination of GA and cisplatin results in an enhancement of apoptosis effects in a time-dependent manner. (A) A549/DDP cells were treated with GA or cisplatin alone or combined with GA $(2 \mu \mathrm{M})$ and cisplatin $(10 \mu \mathrm{g} / \mathrm{mL})$ for 24,48 , and 72 hours. (B) A549/DDP cells were treated with GA or cisplatin alone or combined with GA and cisplatin for 48 hours. The levels of Bax, Bcl-2, caspase-9, and caspase-3 were determined by Western blot analysis. (C and F) The Bax and cleaved caspase-3 protein in cells following the combined treatments was markedly increased compared with the cells exposed to GA or cisplatin alone. (D and E) The Bcl-2 and pro-caspase-9 proteins in cells following the combined treatments were markedly decreased compared with that cells exposed to GA or cisplatin alone. Data are shown as the mean values \pm SD from three independent experiments. $* P<0.05$; $* * P<0.01$; bars, SD.

Abbreviations: GA, gambogic acid; SD, standard deviation; CDDP, cisplatin.

drug accumulation, ${ }^{31}$ enhanced drug inactivation, ${ }^{32}$ restored DNA damage repair, ${ }^{33}$ inactivated apoptotic signaling. ${ }^{34}$ The reducing drug accumulation is on account of drug-resistant proteins. ${ }^{35-38}$ Several studies have shown that reducing cisplatin-resistant protein could reverse cisplatin resistance or enhance tumor sensitivity to cisplatin. ${ }^{39-41} \mathrm{~A}$ host of cisplatinresistant proteins have been reported, for instance, LRP, MRP2, and breast cancer resistance protein. ${ }^{36,42,43} \mathrm{LRP}$ is 

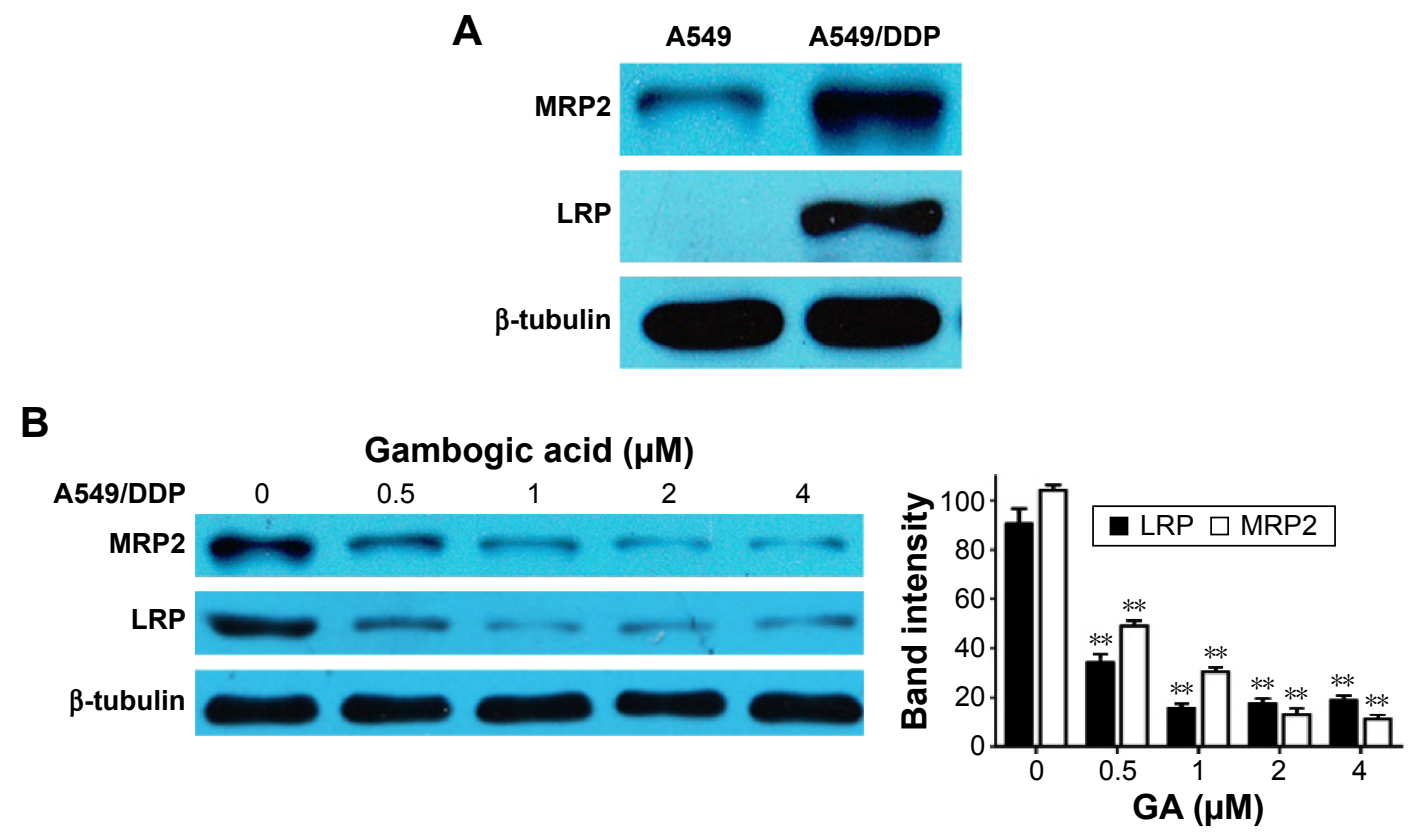

Figure 6 GA downregulates the protein expression of MRP2 and LRP in a dose-dependent way.

Notes: (A) The basic protein expression of MRP2 and LRP in untreated A549 and A549/DDP cells. (B) Cells were treated with GA at the indicated concentrations (0, 0.5 , 1.0, 2.0, and $4.0 \mu \mathrm{M}$ ) for 24 hours. The levels of MRP2 and LRP were determined by Western blot analysis. Data are shown as the mean values \pm SD from three independent experiments. **P<0.0I; bars, SD.

Abbreviations: GA, gambogic acid; LRP, lung resistance protein; MRP2, multidrug resistance-associated protein 2; SD, standard deviation.

overexpressed in several non-P-glycoprotein cancer cell lines with performance of MDR. ${ }^{44} \mathrm{LRP}$ is a small subcellular structure located at cytoplasmatic vaults that may be in charge of subsequent exocytosis of agents from the cell. ${ }^{45}$ In vitro, it has been found that LRP is related to resistance to melphalan, cisplatin, and doxorubicin. ${ }^{44} \mathrm{MRP} 2$ is overexpressed in a lot of cisplatin-resistant cell lines. ${ }^{46,47}$ To figure out that whether the protein could lead to cisplatin resistance or not, we first detected the expression of LRP and MRP2 protein in A549/ DDP and A549 cells. Western blot analysis demonstrated that the expression of LRP and MRP2 protein of A549/DDP cells is significantly more than A549 cells (Figure 6A), which is consistent with the previous researches. ${ }^{48,49}$ Interestingly, GA could significantly downregulate the protein expression level of MRP2 and LRP of A549/DDP cells in a concentrationdependent manner (Figure 6B). Unexpected, there was not the same result on A549 cells. The MRP2 protein expression level of A549 cells was too little to be observed, letting alone LRP protein (Figure 6A). This was probably a consequence of the LRP and MRP2 protein of A549 cells, being too less than A549/DDP cells. For this reason, GA could not fully exert its same effects of downregulating resistance-associated protein on A549 cells. It seems that the phenomenon of the cell viability drop value of A549 cells was lower than A549/ DDP cells after the same treatment sequence of cisplatin alone to GA plus cisplatin on 24 and 48 hours, respectively
(Figure 4A and B). Therefore, GA might enhance the cytotoxic effects of cisplatin by reducing cisplatin-resistant LRP and MRP2 protein on A549/DDP cells.

Excision repair cross-complementing 1 (ERCC1) has become a popular research subject in platinum resistance of NSCLC. ERCC1, as a DNA repair endonuclease, is charge of the $5^{\prime}$-incision in DNA excision repair and recognizing and removing platinum-induced nucleotide adducts in the nucleotide excision repair pathway. ${ }^{50,51}$ ERCC1 overexpression participated in cisplatin resistance by inhibiting the EGFR pathway activation. ${ }^{52}$ These discoveries are in accordance with the clinical phenomenon that ERCC1 in NSCLC prohibits cisplatin efficacy. High ERCC1 of NSCLC tumors will negative respond to cisplatin-based chemotherapy. ${ }^{53,54}$ In this research, we applied the limited theory to explain this phenomenon of GA enhancing the cytotoxic effects of cisplatin on A549/DDP cells by downregulating LRP and MRP2 protein. GA could influence ERCC1 gene or protein expression, thus partially enhancing the cytotoxic effects of cisplatin on A549/DDP cells. Therefore, it is very necessary to confirm this hypothesis in next step.

In conclusion, our results demonstrated that GA has remarkable antigrowth effect by inducing cell cycle arrest and cell apoptosis. Downregulation of cisplatin-resistant protein LRP and MRP2 might represent a mechanism for the effects of GA on cisplatin-resistant human lung cancer A549/DDP cells. 
Combination of GA with cisplatin enhanced the anti-tumor effects on cisplatin-resistant lung cancer cells by downregulating MRP2 and LRP expression. These findings implied that GA may be used as a promising adjuvant drug for the therapy of cisplatin-resistant human lung cancer.

\section{Acknowledgments}

The study was supported by the National Nature Science Foundation of China (81201736); the Science and Technology Planning Project of Guangdong Province, People's Republic of China(KZ1021, KZ0710, 11401S010013, 11401S009015); and the Science and Technology Innovation Project of Guangdong Medical University, People's Republic of China (TD1124).

\section{Disclosure}

The authors report no conflicts of interest in this work.

\section{References}

1. Siegel R, Naishadham D, Jemal A. Cancer statistics, 2012. CA Cancer J Clin. 2012;62(1):10-29.

2. D'Addario G, Pintilie M, Leighl NB, Feld R, Cerny T, Shepherd FA. Platinum-based versus non-platinum-based chemotherapy in advanced non-small-cell lung cancer: a meta-analysis of the published literature. J Clin Oncol. 2005;23(13):2926-2936.

3. Yang Y, Li H, Hou S, Hu B, Liu J, Wang J. The noncoding RNA expression profile and the effect of lncRNA AK126698 on cisplatin resistance in non-small-cell lung cancer cell. PLoS One. 2013;8(5):e65309.

4. Huang H, Chen D, Li S, et al. Gambogic acid enhances proteasome inhibitor-induced anticancer activity. Cancer Lett. 2011;301(2): 221-228.

5. Yang LJ, Chen Y. New targets for the antitumor activity of gambogic acid in hematologic malignancies. Acta Pharmacol Sin. 2013;34(2): 191-198.

6. Wang LH, Yang JY, Yang SN, et al. Suppression of NF-kappaB signaling and P-glycoprotein function by gambogic acid synergistically potentiates adriamycin-induced apoptosis in lung cancer. Curr Cancer Drug Targets. 2014;14(1):91-103.

7. Ortiz-Sanchez E, Daniels TR, Helguera G, Martinez-Maza O, Bonavida B, Penichet ML. Enhanced cytotoxicity of an anti-transferrin receptor IgG3avidin fusion protein in combination with gambogic acid against human malignant hematopoietic cells: functional relevance of iron, the receptor, and reactive oxygen species. Leukemia. 2009;23(1):59-70.

8. Zhang H, Lei Y, Yuan P, et al. ROS-mediated autophagy induced by dysregulation of lipid metabolism plays a protective role in colorectal cancer cells treated with gambogic acid. PLoS One. 2014;9(5):e96418.

9. Yi T, Yi Z, Cho SG, et al. Gambogic acid inhibits angiogenesis and prostate tumor growth by suppressing vascular endothelial growth factor receptor 2 signaling. Cancer Res. 2008;68(6):1843-1850.

10. Nie F, Zhang X, Qi Q, et al. Reactive oxygen species accumulation contributes to gambogic acid-induced apoptosis in human hepatoma SMMC-7721 cells. Toxicology. 2009;260(1-3):60-67.

11. Li C, Qi Q, Lu N, et al. Gambogic acid promotes apoptosis and resistance to metastatic potential in MDA-MB-231 human breast carcinoma cells. Biochem Cell Biol. 2012;90(6):718-730.

12. Zhao Q, Yang Y, Yu J, et al. Posttranscriptional regulation of the telomerase hTERT by gambogic acid in human gastric carcinoma 823 cells. Cancer Lett. 2008;262(2):223-231.

13. Wen C, Huang L, Chen J, et al. Gambogic acid inhibits growth, induces apoptosis, and overcomes drug resistance in human colorectal cancer cells. Int J Oncol. 2015;47(5):1663-1671.
14. Ishaq M, Khan MA, Sharma K, Sharma G, Dutta RK, Majumdar S. Gambogic acid induced oxidative stress dependent caspase activation regulates both apoptosis and autophagy by targeting various key molecules (NF-kappaB, Beclin-1, p62 and NBR1) in human bladder cancer cells. Biochim Biophys Acta. 2014;1840(12):3374-3384.

15. Li D, Yang H, Li R, et al. Antitumor activity of gambogic acid on NCI-H1993 xenografts via MET signaling pathway downregulation. Oncol Lett. 2015;10(5):2802-2806.

16. Shi X, Chen X, Li X, et al. Gambogic acid induces apoptosis in imatinib-resistant chronic myeloid leukemia cells via inducing proteasome inhibition and caspase-dependent bcr-abl downregulation. Clin Cancer Res. 2014;20(1):151-163.

17. Wang $\mathrm{X}, \mathrm{Lu} \mathrm{N}$, Yang Q, et al. Studies on chemical modification and biology of a natural product, gambogic acid (III): determination of the essential pharmacophore for biological activity. Eur J Med Chem. 2011;46(4):1280-1290.

18. Chi Y, Zhan XK, Yu H, et al. An open-labeled, randomized, multicenter phase IIa study of gambogic acid injection for advanced malignant tumors. Chin Med J (Engl). 2013;126(9):1642-1646.

19. Zhao W, You CC, Zhuang JP, et al. Viability inhibition effect of gambogic acid combined with cisplatin on osteosarcoma cells via mitochondriaindependent apoptotic pathway. Mol Cell Biochem. 2013;382(1-2): $243-252$.

20. Wang LH, Li Y, Yang SN, et al. Gambogic acid synergistically potentiates cisplatin-induced apoptosis in non-small-cell lung cancer through suppressing NF-kappaB and MAPK/HO-1 signalling. Br J Cancer. 2014; 110(2):341-352.

21. Yang Y, Sun X, Yang Y, et al. Gambogic acid enhances the radiosensitivity of human esophageal cancer cells by inducing reactive oxygen species via targeting Akt/mTOR pathway. Tumour Biol. 2015;37(2):1853-1862.

22. Yang M, Yang Y, Cui H, et al. The natural compound gambogic acid radiosensitizes nasopharyngeal carcinoma cells under hypoxic conditions. Tumori. 2015;102(2):135-143.

23. Wang T, Wei J, Qian X, Ding Y, Yu L, Liu B. Gambogic acid, a potent inhibitor of survivin, reverses docetaxel resistance in gastric cancer cells. Cancer Lett. 2008;262(2):214-222.

24. Wang S, Wang L, Chen M, Wang Y. Gambogic acid sensitizes resistant breast cancer cells to doxorubicin through inhibiting P-glycoprotein and suppressing survivin expression. Chem Biol Interact. 2015;235:76-84.

25. Wang J, Yuan Z. Gambogic acid sensitizes ovarian cancer cells to doxorubicin through ROS-mediated apoptosis. Cell Biochem Biophys. 2013; 67(1):199-206.

26. Wang X, Deng R, Lu Y, et al. Gambogic acid as a non-competitive inhibitor of ATP-binding cassette transporter B1 reverses the multidrug resistance of human epithelial cancers by promoting ATP-binding cassette transporter B1 protein degradation. Basic Clin Pharmacol Toxicol. 2013; 112(1):25-33.

27. Sakamoto M, Kondo A, Kawasaki K, et al. Analysis of gene expression profiles associated with cisplatin resistance in human ovarian cancer cell lines and tissues using cDNA microarray. Hum Cell. 2001;14(4): 305-315.

28. Li H, Chen X, Yu Y, et al. Metformin inhibits the growth of nasopharyngeal carcinoma cells and sensitizes the cells to radiation via inhibition of the DNA damage repair pathway. Oncol Rep. 2014;32(6): 2596-2604.

29. Siddik ZH. Cisplatin: mode of cytotoxic action and molecular basis of resistance. Oncogene. 2003;22(47):7265-7279.

30. Tian L, Liu J, Chen BA, et al. [Reversal effect of gambogic acid on multidrug resistance of K562/A02 cell line]. Zhongguo Shi Yan Xue Ye Xue Za Zhi. 2012;20(2):252-257.

31. Amable L, Fain J, Gavin E, Reed E. Gli1 contributes to cellular resistance to cisplatin through altered cellular accumulation of the drug Oncol Rep. 2014;32(2):469-474.

32. Koch M, Krieger ML, Stölting D, et al. Overcoming chemotherapy resistance of ovarian cancer cells by liposomal cisplatin: molecular mechanisms unveiled by gene expression profiling. Biochem Pharmacol. 2013;85(8):1077-1090. 
33. Hubaux R, Thu KL, Vucic EA, et al. Microtubule affinity-regulating kinase 2 is associated with DNA damage response and cisplatin resistance in non-small cell lung cancer. Int J Cancer. 2015;137(9):2072-2082.

34. Duan S, Tsai Y, Keng P, Chen Y, Lee SO, Chen Y. IL-6 signaling contributes to cisplatin resistance in non-small cell lung cancer via the up-regulation of anti-apoptotic and DNA repair associated molecules. Oncotarget. 2015;6(29):27651-27660.

35. Korita PV, Wakai T, Shirai Y, et al. Multidrug resistance-associated protein 2 determines the efficacy of cisplatin in patients with hepatocellular carcinoma. Oncol Rep. 2010;23(4):965-972.

36. Yamasaki M, Makino T, Masuzawa T, et al. Role of multidrug resistance protein 2 (MRP2) in chemoresistance and clinical outcome in oesophageal squamous cell carcinoma. Br J Cancer. 2011;104(4):707-713.

37. Yoh K, Ishii G, Yokose T, et al. Breast cancer resistance protein impacts clinical outcome in platinum-based chemotherapy for advanced nonsmall cell lung cancer. Clin Cancer Res. 2004;10(5):1691-1697.

38. Zhang B, Liu M, Tang HK, et al. The expression and significance of MRP1, LRP, TOPOIIbeta, and BCL2 in tongue squamous cell carcinoma. J Oral Pathol Med. 2012;41(2):141-148.

39. Wang CK, Zhang Y, Zhang ZJ, Qiu QW, Cao JG, He ZM. Effects of VBMDMP on the reversal of cisplatin resistance in human lung cancer A549/DDP cells. Oncol Rep. 2015;33(1):372-382.

40. Wang TH, Wan JY, Gong X, Li HZ, Cheng Y. Tetrandrine enhances cytotoxicity of cisplatin in human drug-resistant esophageal squamous carcinoma cells by inhibition of multidrug resistance-associated protein 1. Oncol Rep. 2012;28(5):1681-1686.

41. Li S, Lei Y, Jia Y, Li N, Wink M, Ma Y. Piperine, a piperidine alkaloid from Piper nigrum re-sensitizes P-gp, MRP1 and BCRP dependent multidrug resistant cancer cells. Phytomedicine. 2011;19(1):83-87.

42. Lehmann T, Torky AR, Stehfest E, Hofmann S, Foth H. Expression of lung resistance-related protein, LRP, and multidrug resistance-related protein, MRP1, in normal human lung cells in long-term cultures. Arch Toxicol. 2005;79(10):600-609.

43. Bharthuar A, Saif URS, Black JD, et al. Breast cancer resistance protein (BCRP) and excision repair cross complement-1 (ERCC1) expression in esophageal cancers and response to cisplatin and irinotecan based chemotherapy. J Gastrointest Oncol. 2014;5(4):253-258.
44. Izquierdo MA, Shoemaker RH, Flens MJ, et al. Overlapping phenotypes of multidrug resistance among panels of human cancer-cell lines. Int J Cancer. 1996;65(2):230-237.

45. Scheffer GL, Wijngaard PL, Flens MJ, et al. The drug resistance-related protein LRP is the human major vault protein. Nat Med. 1995;1(6): $578-582$.

46. Taniguchi K, Wada M, Kohno K, et al. A human canalicular multispecific organic anion transporter (cMOAT) gene is overexpressed in cisplatin-resistant human cancer cell lines with decreased drug accumulation. Cancer Res. 1996;56(18):4124-4129.

47. Kool M, de Haas M, Scheffer GL, et al. Analysis of expression of cMOAT (MRP2), MRP3, MRP4, and MRP5, homologues of the multidrug resistance-associated protein gene (MRP1), in human cancer cell lines. Cancer Res. 1997;57(16):3537-3547.

48. Cao Y, Xia QH, Meng H, Zhong AP. Pharmacological effects of serum containing Chinese medicine Bushen Huayu Jiedu Compound Recipe in lung cancer drug-resistance cells. Chin J Integr Med. 2008;14(1): 46-50.

49. Torky AR, Stehfest E, Viehweger K, Taege C, Foth H. Immunohistochemical detection of MRPs in human lung cells in culture. Toxicology. 2005;207(3):437-450.

50. Ma XJ, Shang L, Zhang WM, Wang MR, Zhan QM. Mitotic regulator Nlp interacts with XPA/ERCC1 complexes and regulates nucleotide excision repair (NER) in response to UV radiation. Cancer Lett. 2016; 373(2):214-221.

51. Zamble DB, Mu D, Reardon JT, Sancar A, Lippard SJ. Repair of cisplatin-DNA adducts by the mammalian excision nuclease. Biochemistry-US. 1996;35(31):10004-10013.

52. Lian S, Su H, Zhao BX, Liu WY, Zheng LW, Miao JY. Synthesis and discovery of pyrazole-5-carbohydrazide N-glycosides as inducer of autophagy in A549 lung cancer cells. Bioorg Med Chem. 2009;17(20): 7085-7092.

53. Olaussen KA, Dunant A, Fouret P, et al. DNA repair by ERCC1 in non-small-cell lung cancer and cisplatin-based adjuvant chemotherapy. N Engl J Med. 2006;355(10):983-991.

54. Soria JC. ERCC1-tailored chemotherapy in lung cancer: the first prospective randomized trial. J Clin Oncol. 2007;25(19):2648-2649.
OncoTargets and Therapy

\section{Publish your work in this journal}

OncoTargets and Therapy is an international, peer-reviewed, open access journal focusing on the pathological basis of all cancers, potential targets for therapy and treatment protocols employed to improve the management of cancer patients. The journal also focuses on the impact of management programs and new therapeutic agents and protocols on

\section{Dovepress}

patient perspectives such as quality of life, adherence and satisfaction The manuscript management system is completely online and includes a very quick and fair peer-review system, which is all easy to use. Visit http://www.dovepress.com/testimonials.php to read real quotes from published authors. 